Tecnología y

Ciencias $\approx$ Agua

DOI: $10.24850 / j-t y c a-2021-02-11$

Artículos

\title{
Evapotranspiración e intercambio de energía en un bosque templado de México
}

\section{Evapotranspiration and energy exchange in a temperate forest in Mexico}

Melissa Hidalgo-Sánchez ${ }^{1}$, ORCID: https://orcid.org/0000-0003-21626726

Gregorio Ángeles-Pérez², ORCID: https://orcid.org/0000-0002-95502825

Enrico A. Yépez³, ORCID: https://orcid.org/0000-0003-4746-573X

Francisca Ofelia Plascencia-Escalante ${ }^{4}$, ORCID: https://orcid.org/00000002-9475-5716

Josué Delgado-Balbuena5, ORCID: https://orcid.org/0000-0001-79281869

Teresa Margarita González-Martínez6, ORCID: https://orcid.org/00000003-1629-5184

${ }^{1}$ Colegio de Postgraduados, Texcoco, México, hidalgo.melissa@colpos.mx ${ }^{2}$ Colegio de Postgraduados, Texcoco, México, gangeles@colpos.mx 3Instituto Tecnológico de Sonora, Cd. Obregón, México, yepezglz@gmail.com 
${ }^{4}$ Colegio de Postgraduados, Texcoco, México, fplascen@colpos.mx

${ }^{5}$ Instituto Nacional de Investigaciones Forestales, Agrícolas y Pecuarias, Ojuelos de Jalisco, México, josuedlb@gmail.com

${ }^{6}$ Colegio de Postgraduados, Texcoco, México, teresa.gonzalez@colpos.mx

Autor para correspondencia: Gregorio Ángeles-Pérez, gangeles@colpos.mx

\section{Resumen}

En los ecosistemas forestales, el balance de energía, el ciclo del agua y el ciclo del carbono se vinculan a través del proceso de evapotranspiración. La evapotranspiración es altamente variable en espacio y tiempo, y está controlada por numerosos factores biofísicos. El objetivo de este estudio fue analizar la variabilidad estacional de la evapotranspiración e identificar los principales factores que influyen en dicho proceso. El método de eddy covariance se utilizó para medir los flujos de calor sensible y calor latente (equivalente a evapotranspiración) en un bosque bajo manejo forestal, ubicado en la región forestal de Zacualtipán, Hidalgo. Simultáneamente se midió la radiación neta, el flujo de calor del suelo, el contenido de agua en el suelo y otros parámetros meteorológicos. A lo largo del periodo analizado, la proporción de la radiación neta con los flujos de calor latente y calor sensible fue similar la mayor parte del tiempo. La evapotranspiración total anual observada fue de $980 \mathrm{~mm}$. Ésta fue menor en el periodo de "nortes" y no se encontraron diferencias significativas entre el periodo de lluvias y el de secas. La temperatura, humedad del 


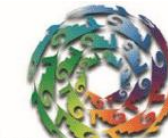

Tienoncias

suelo, radiación neta y déficit de presión de vapor explicaron la mayor parte de la variabilidad de la evapotranspiración. Se recomienda realizar estudios posteriores que involucren el análisis de la productividad del ecosistema y eficiencia en el uso del agua, para comprender mejor los procesos del ecosistema e identificar prácticas apropiadas para el manejo forestal sostenible.

Palabras clave: balance de energía, bosque templado, calor latente, covarianza de vórtices, variación estacional, Pinus patula.

\section{Abstract}

In forest ecosystems the energy balance, the water cycle and the carbon cycle are linked through the evapotranspiration process. Evapotranspiration is highly variable in space and time since it is controlled by numerous biophysical factors. The objective of this study was to analyze the seasonal variability of evapotranspiration and identify the main factors that influence this process. The eddy covariance technique was used to measure fluxes of sensible heat and latent heat (equivalent to evapotranspiration) in a temperate forest under forest management, located in the region of Zacualtipán, Hidalgo, Mexico. Simultaneously, net radiation, soil heat flux, soil moisture content and other meteorological parameters were measured. During the analyzed period, the proportion of the net radiation with the latent heat and sensible heat fluxes was similar most of the time. The total annual evapotranspiration observed was $980 \mathrm{~mm}$. It was lower in the "cold front" season and no differences were found between the rainy season and the 
dry season. Temperature, soil moisture, net radiation and vapor pressure deficit explained most of the variability of evapotranspiration. Further studies are necessary to involve the analysis of ecosystem productivity and water use efficiency, to better understand ecosystem processes and identify appropriate sustainable forest management practices.

Keywords: Energy balance, temperate forest, latent heat, eddy covariance, seasonal variation, Pinus patula.

Recibido: $10 / 02 / 2020$

Aceptado: $22 / 07 / 2020$

\section{Introducción}

Los bosques juegan un papel importante en el equilibrio hídrico y energético de la superficie terrestre (Dolman, Moors, Grundwald, \& Bernhofer, 2003; Matsumoto et al., 2008), ya que influyen en los componentes del ciclo hidrológico, y tienen la capacidad de capturar y redistribuir la energía solar (Ellison et al., 2017). De la energía solar que 
llega a la superficie terrestre una parte es reflejada hacia la atmósfera, el resto queda disponible en la superficie para impulsar los procesos naturales de los ecosistemas, y se le conoce como radiación neta (Huryna \& Pokorný, 2016). La radiación neta se distribuye en flujo de calor sensible, flujo de calor del suelo y flujo de calor latente. Este último proporciona la energía para que el proceso de evapotranspiración se realice (Bonan, 2016).

La evapotranspiración es la suma de vapor de agua liberada por el suelo y la vegetación hacia la atmósfera (Law et al., 2002; Biederman et al., 2016). Gran parte de dicho proceso está directamente asociado con la productividad del ecosistema, pues la asimilación de $\mathrm{CO}_{2}$, durante la fotosíntesis y la transpiración, está regulada por la dinámica de apertura y cierre de los estomas (Canny, 1998). En los bosques, la evapotranspiración está compuesta por evaporación del suelo, interceptación del dosel y transpiración de las plantas (Sun, Domec, \& Amatya, 2016).

La evapotranspiración varía con el clima, tipo de vegetación y etapa fenológica, y se ve directamente afectada por las estrategias de manejo de la tierra y el cambio climático (Liu \& El-Kassaby, 2018). Por lo tanto, los cambios en la cobertura del suelo y los factores del clima afectan directamente el ciclo hidrológico regional, el balance de energía y las funciones del ecosistema, incluyendo la evapotranspiración (Liu et al., 2018).

El manejo de los recursos forestales en México se ha orientado a desarrollar practicas silvícolas que permitan maximizar la producción de madera (Perez-Verdin, Monarrez-González, Tecle, y Pompa-Garcia, 
2018). Estas prácticas mejoran la productividad de los ecosistemas y contribuyen a la disminución de gases de efecto invernadero (Ussiri \& Lal, 2017). Sin embargo, varios estudios han reportado que la implementación de estas actividades causa un impacto negativo en la regulación de los flujos de agua (Monarrez-González, Perez-Verdin, López-González, Márquez-Linarez, \& González-Elizondo, 2018).

A pesar de la importancia de entender la evapotranspiración de los bosques, las mediciones directas a escala de paisaje sólo han sido posibles en las últimas dos décadas (Baldocchi, 2019; Wilson \& Baldocchi, 2000) y en los últimos años para el caso de México (Delgado-Balbuena et al., 2019). Generalmente las estimaciones de evapotranspiración, en ecosistemas forestales, han sido derivadas como un residuo del balance hídrico (Özhan, Gökbulak, Serengil, \& Özcan, 2010). También es común que la evapotranspiración se considere una proporción de la evapotranspiración potencial y, por lo tanto, se calcule a partir de información meteorológica obtenida en estaciones fijas con numerosos métodos directos, teóricos y empíricos (García, Campos, Di Bella, \& Posse, 2013). Estos enfoques tienden a ser relativamente económicos; sin embargo, las estimaciones de evapotranspiración derivadas tienen una capacidad limitada para informar sobre los controles biofísicos en escalas de tiempo más cortas. Actualmente existen otros métodos más sofisticados y de mayor precisión, que se basan en el análisis de flujos turbulentos para determinar la evapotranspiración real, entre ellos el método de eddy covariance (García et al., 2013). Este método proporciona mediciones directas y continuas del intercambio de energía y vapor de agua entre un ecosistema y la atmósfera (Baldocchi, 2003; Aubinet, Vesala, \& Papale, 2012). 
En el ámbito global, diversos estudios que han utilizado este método han informado que el reparto de energía y la evapotranspiración tienen variaciones estacionales (Mo, Liu, Chen, y Hu, 2018; Sanwangsri, Hanpattanakit, \& Chidthaisong, 2017; Tsuruta, Kosugi, Takanashi, \& Tani, 2016); interanuales (Miyazaki et al., 2014), y entre ecosistemas (Brümmer et al., 2012; Cristiano et al., 2015; Sun, Zou, Wilcox, \& Stebler, 2018). En México, los estudios realizados sobre el intercambio de agua y energía entre los ecosistemas terrestres y la atmósfera son limitados. En su mayoría se centran en matorrales y bosques tropicales caducifolios en las zonas áridas y semiáridas del país (Martínez-Yrízar, Álvarez-Sánchez, \& Maass, 2017). La mayoría de estas investigaciones se han centrado en tratar de comprender la influencia de la precipitación (Pérez-Ruiz et al., 2010); factores topográficos (Berry, Gotsch, Holwerda, Muñoz-Villers, \& Asbjornsen, 2016); diferentes coberturas de vegetación (Holwerda, Alvarado-Barrientos, \& González-Martínez, 2016), y el papel de la neblina (Alvarado-Barrientos, Holwerda, Asbjornsen, Dawson, \& Bruijnzeel, 2014) en la dinámica hidrológica y el intercambio de energía. En el caso de bosques templados, este estudio es el primer reporte en México de intercambio de energía y evapotranspiración, con base en mediciones continuas de flujos turbulentos. Comprender la variabilidad de estos procesos y sus principales mecanismos de control proporcionará bases sólidas para abordar los desafíos del cambio climático y los efectos de las decisiones de manejo forestal. Así, los objetivos planteados en este trabajo fueron: (a) estimar el intercambio de energía y la evapotranspiración en un bosque bajo manejo forestal con el método de eddy covariance; (b) evaluar qué variables meteorológicas controlan la 
evapotranspiración; y (c) analizar la variabilidad estacional de los flujos de agua y energía en un bosque templado del centro de México.

\section{Materiales y métodos}

\section{Área de estudio}

El área de estudio forma parte del Sitio de Monitoreo Intensivo de Carbono Atopixco $\left(20^{\circ} 37^{\prime} 49.78^{\prime \prime} \mathrm{N}\right.$ y $98^{\circ} 37^{\prime} 51.01^{\prime \prime} \mathrm{W}$, y $20^{\circ} 35^{\prime} 18.74^{\prime \prime} \mathrm{N}$ y $98^{\circ}$ 35' 23" W), ubicado en la región forestal de Zacualtipán, Hidalgo, México (Figura 1). Es un bosque templado bajo manejo forestal para producción maderable desde 1980. En este sitio se utiliza el método de desarrollo silvícola de regeneración de árboles padre, el cual ha permitido generar rodales coetáneos dominados por Pinus patula Schltdl. et Cham. También forman parte de la estructura especies como Quercus laurina Humb. et Bonpl; Quercus rugosa Neé; Quercus excelsa Liebm; Clethra mexicana D.C., y Vaccinium leucanthum Schltdl en bajas densidades (Ángeles-Pérez et al., 2015). 
Tecnología y

Ciencias $\approx$ Agua
2021, Instituto Mexicano de Tecnología del Agua

Open Access bajo la licencia CC BY-NC-SA 4.0

(https://creativecommons.org/licenses/by-nc-sa/4.0/)
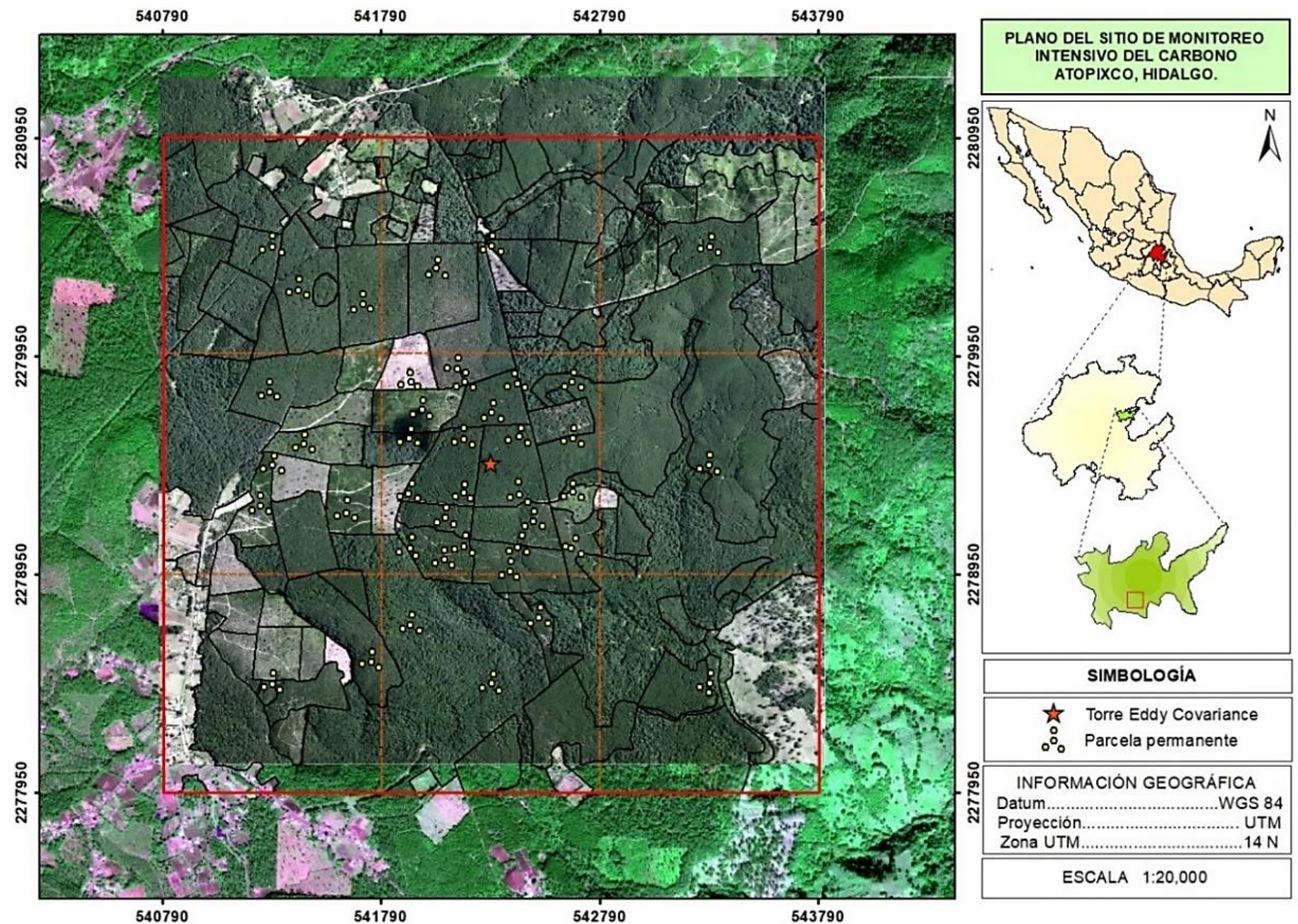

Figura1. Ubicación del sitio de estudio.

El clima predominante de la región es templado-húmedo, con una estación marcada de lluvias entre junio y octubre (1 200-1 600 mm anuales); y temperatura media anual que oscila entre 12.5 y $14.5{ }^{\circ} \mathrm{C}$ (Soriano-Luna et al., 2018). Por localizarse en una de las partes más altas de la Sierra Madre Oriental (2 075 msnm) (Ángeles-Pérez et al., 2015), dicho ecosistema se encuentra directamente expuesto a los vientos húmedos provenientes del Golfo de México y a la entrada de frentes fríos 
("nortes") que dominan durante la temporada de invierno. Los tipos de suelo son feozem háplico $(\mathrm{Hh})$, profundos y ricos en materia orgánica (Aguirre-Salado et al., 2009).

El Sitio de Monitoreo Intensivo de Carbono se estableció de acuerdo con la metodología propuesta por Hollinger (2008). El diseño consistió en un polígono de $3 \times 3 \mathrm{~km}$, dividido en 9 cuadrantes de $1 \mathrm{~km}^{2}$ cada uno, con parcelas de muestreo permanentes, similares a las utilizadas por la Comisión Nacional Forestal (Conafor, 2012) en el Inventario nacional forestal y de suelos. En el centro del polígono se instrumentó una torre de flujo eddy covariance para medir el intercambio de energía y vapor de agua entre el ecosistema y la atmósfera (Figura 1).

\section{Sistema eddy covariance y sensores meteorológicos}

El sistema de eddy covariance se instaló en una torre, a $32 \mathrm{~m}$ sobre el nivel del suelo. Se utilizó un analizador de gas al infrarrojo de vía abierta (IRGA, modelo LI-7500A, LI-COR, Lincoln, NE, EUA) para medir la concentración de vapor de agua, y un anemómetro sónico tridimensional (Gill Windmaster Pro) para medir la velocidad $(\mathrm{m} / \mathrm{s}$ ) y dirección del viento $\left(^{\circ}\right)$ en sus tres componentes $x, y, z$. Ambos equipos midieron a una frecuencia de $10 \mathrm{~Hz}$ y los datos se registraron en una interface controladora y de almacenamiento (LI-7550, Li-COR, Lincoln, NE, EUA). 
Tecnología y

Ciencias $\stackrel{\Xi}{\triangleleft}$ gua

Simultáneamente se midieron variables meteorológicas: temperatura del aire y humedad relativa a $31 \mathrm{~m}$ sobre el suelo (sensor Vaisala HUMICAP); radiación global, radiación neta y radiación fotosintéticamente activa a una altura de $33 \mathrm{~m}$ (piranómetro LI-COR LI200, radiómetro neto CNR4 Kip \& Zonen y sensor quantum LI-COR LI190R, respectivamente), y precipitación por encima de la superficie del dosel (pluviómetro TR-525Mrain)

Para medir la temperatura del suelo, se instalaron dos termopares (TCAV Campbell Scientific) a $5 \mathrm{~cm}$ por debajo de la superficie del suelo. El contenido de agua en el suelo se midió con dos reflectómetros (CS616 Campbell Scientific). El flujo del calor del suelo se midió en cuatro puntos con placas de calor del suelo (HFP01 Campbell Scientific) enterradas a 7 $\mathrm{cm}$ de la superficie del suelo.

La información meteorológica y de las condiciones del suelo se almacenó en intervalos de 30 minutos, en un registrador de datos CR3000 (Campbell Scientific). El periodo de colecta de datos abarcó del $1^{\circ}$ de octubre de 2017 al 30 de septiembre de 2018 .

\section{Procesamiento de datos}

Los datos colectados fueron procesados en el software Eddy Pro (LI-COR, Inc., 2016) versión 6.2.0. Este software es una aplicación desarrollada por LI-COR utilizada para calcular los flujos de calor sensible y latente en 
periodos de 30 minutos, a través de la covarianza de la velocidad del viento, con la concentración de vapor de agua y la temperatura sónica, respectivamente (Burba, 2013).

El procedimiento incluyó la remoción de picos de la serie de tiempo (Vickers \& Mahrt, 1997), corrección "W-boost" del error de cálculo del anemómetro que subestima la velocidad vertical del viento; corrección de la inclinación con el método de doble rotación de coordenadas (Wilczak, Oncley, \& Stage, 2001); corrección de retrasos entre las señales del analizador de gas y el anemómetro; correcciones por fluctuaciones en la densidad del aire (Burba, 2013; Webb, Pearman, \& Leuning, 1980); y correcciones espectrales de alta y baja frecuencia (Moncrieff, 1997; Moncrieff, 2004). Finalmente, con el software se calcularon los indicadores de control de calidad para todos los flujos con base en la prueba de condiciones turbulentas y de estado estable (Mauder \& Foken, 2006). El resultado del procesamiento de datos fue una base datos, la cual incluyó el valor de las variables intermedias y finales calculadas para cada periodo.

\section{Control de calidad de datos}

Este procedimiento se realizó en la base de datos que resultó del procesamiento en Eddy Pro. La base de datos muestra el valor de los flujos de calor sensible $(H)$, calor latente (LE) y el flujo de agua, 
acompañado de un código numérico $(0,1 \circ 2)$, que indica la calidad de los flujos. El valor "0" indica flujos de mejor calidad, "1" representa los flujos de buena calidad, mientras que el "2" señala los flujos de mala calidad. Los flujos considerados de mala calidad fueron eliminados. También se eliminaron los registros que se encontraban fuera del límite de magnitud de flujo y aquellos valores que fueron registrados en periodos de intensa precipitación.

\section{Relleno de datos (Gapfilling)}

A pesar de que la técnica de eddy covariance permitió la generación de mediciones casi continuas a lo largo del tiempo, hubo periodos con ausencia de registros causados por fallas en el sistema y por la exclusión de datos de acuerdo con el control de calidad de datos. El relleno de datos generalmente se realiza a través de la correlación de los flujos con variables meteorológicas (radiación, temperatura o humedad); sin embargo, durante 27 días distribuidos a lo largo del periodo no hubo registros de dichas variables. De tal manera que la estimación de los datos de flujo faltantes se realizó con el algoritmo de muestreo de distribución marginal en REddyProc (Wutzler et al., 2018). Esta herramienta en línea (https://www.bgc-jena.mpg.de/REddyProc/brew/REddyProc.rhtml), además de considerar la covarianza de flujos con variables meteorológicas (Falge, Baldocchi, \& Olson, 2001), permite la autocorrelación temporal de los 
flujos (Reichstein et al., 2005). El porcentaje de datos que se generaron con relación al total fue del $39 \%$, de los cuales el $12 \%$ correspondió al periodo diurno y el $27 \%$ al periodo nocturno.

\section{Cierre de balance de energía}

La evaluación del cierre de balance de energía es un procedimiento estandarizado dentro de la metodología eddy covariance para verificar la calidad de las mediciones. Este procedimiento consistió en graficar los valores de 30 minutos de la suma de los flujos de calor sensible $(\mathrm{H})$ y calor latente (LE) contra la diferencia de los valores de radiación neta ( $R n)$ y el flujo de calor del suelo (G). Se ajustó una línea de tendencia donde el valor de la pendiente y la intercepción de la línea de regresión cuantificaron la confiabilidad del cierre de balance de energía.

\section{Área de influencia (footprint)}

El área de influencia se obtuvo con las medidas de eddy covariance. El análisis se realizó con la herramienta en línea Flux Footprint Prediction (FFP) online data processing (Kljun, Calanca, Rotach, \& Schmid, 2015). 
En un análisis mensual, se observó que el área de influencia se mantuvo constante a lo largo del año.

\section{Análisis de datos}

Para identificar los controles ambientales que influyen en la variación de la evapotranspiración se utilizó una matriz de correlación de Spearman; se eligió este análisis no paramétrico debido a que los datos no presentaron una distribución normal. Las variables utilizadas fueron las siguientes: radiación neta, radiación fotosintéticamente activa, temperatura del aire y del suelo, humedad relativa, déficit de presión de vapor, precipitación y contenido de agua en el suelo. Posteriormente se generó un modelo de regresión lineal múltiple para evaluar la influencia que tienen en conjunto los predictores antes descritos sobre la evapotranspiración; la variable dependiente se normalizó con la transformación de Box-Cox. La confiabilidad del modelo fue evaluada con el coeficiente de determinación ajustado $\left(R^{2}\right.$ adj $)$, error estándar residual (RSE) y el Criterio de Información de Akaike (AIC).

Se analizó la variación estacional de la evapotranspiración en tres periodos: entrada de frentes fríos (noviembre-febrero); época de secas (marzo-mayo), y periodo de lluvias (junio-octubre). Para determinar si existían diferencias estadísticamente significativas entre las temporadas, se utilizó la prueba de Kruskal-Wallis, seguida de un análisis post hoc con 
la prueba de Mann-Whitney-Wilcoxon para determinar qué periodos diferían entre sí. Los análisis estadísticos mencionados se realizaron con el software $R$ Core Team (2018) versión 3.5.0.

\section{Resultados}

\section{Condiciones meteorológicas}

La radiación global, radiación neta y radiación fotosintéticamente activa alcanzaron su punto máximo en el mes de mayo y el mínimo en octubre; en el caso de la radiación neta, el mínimo fue en diciembre (Tabla 1). La temperatura del aire varió de 2 a $20.8^{\circ} \mathrm{C}$ (promedio diario), y durante los meses de junio a septiembre se mantuvo entre los 12 y $17^{\circ} \mathrm{C}$ (Figura 2). La temperatura mínima del aire ocurrió en diciembre y la máxima en mayo. La temperatura del suelo osciló entre 8.5 y $16.8^{\circ} \mathrm{C}$; en general, tuvo una tendencia similar a la temperatura del aire. El déficit de presión de vapor se mantuvo alto durante los meses de marzo, abril y mayo, por lo que la demanda evaporativa fue mayor en este periodo. Todos los 
meses presentaron precipitación; en total se acumularon 953 mm, 20\% menos que lo ocurrido en años anteriores. El $72 \%$ de la precipitación total ocurrió de junio a octubre; octubre tuvo mayor contribución (294.9 $\mathrm{mm}$ ). El contenido de agua en el suelo varió con la precipitación. Los eventos de precipitación intensos a finales del mes de enero provocaron un aumento abrupto en el contenido de agua del suelo para el mes de febrero (Figura 2). La precipitación anual registrada en el sitio de estudio para los años de 2014-2018 fue de 1 255, 812, 1227,1286 y 751 mm, respectivamente.

Tabla1. Valores medios mensuales de evapotranspiración y variables meteorológicas durante el periodo de estudio.

\begin{tabular}{|c|c|c|c|c|c|c|c|c|c|c|c|c|}
\hline \multirow{2}{*}{ Variable } & \multicolumn{3}{|c|}{2017} & \multicolumn{9}{|c|}{2018} \\
\hline & Oct & Nov & Dic & Ene & Feb & Mar & Abr & May & Jun & Jul & Ago & Sep \\
\hline $\begin{array}{c}\mathrm{Rg} \\
\left(\mathrm{W} / \mathrm{m}^{2}\right)\end{array}$ & 136.1 & 175.9 & 156.8 & 144.7 & 171.5 & 201.1 & 210.2 & 229.9 & 175.4 & 215.7 & 180.5 & 164.8 \\
\hline $\begin{array}{c}\mathrm{Rn} \\
\left(\mathrm{W} / \mathrm{m}^{2}\right)\end{array}$ & 99.0 & 115.5 & 95.7 & 99.5 & 121.2 & 144.8 & 151.6 & 164.7 & 128.5 & 159.1 & 126.1 & 130.5 \\
\hline $\begin{array}{c}\text { RFA } \\
\left(\mu \mathrm{mol} / \mathrm{m}^{2} / \mathrm{s}\right)\end{array}$ & 250.4 & 322.6 & 288.0 & 268.3 & 325.8 & 383.8 & 404.5 & 447.1 & 349.5 & 431.9 & 346.2 & 343.8 \\
\hline $\begin{array}{l}\text { Ta promedio } \\
\left({ }^{\circ} \mathrm{C}\right)\end{array}$ & 12.8 & 11.8 & 10.1 & 7.4 & 12.4 & 13.5 & 14.4 & 15.6 & 15.0 & 14.7 & 14.1 & 14.5 \\
\hline $\begin{array}{c}\text { Ta mínima } \\
\left({ }^{\circ} \mathrm{C}\right)\end{array}$ & 3.8 & 1.7 & -4.7 & -0.4 & 6.8 & 4.8 & 3.9 & 9.2 & 10.7 & 9.8 & 10.5 & 10.7 \\
\hline
\end{tabular}


Ciencias $\approx$ Agua

2021, Instituto Mexicano de Tecnología del Agua

Open Access bajo la licencia CC BY-NC-SA 4.0

(https://creativecommons.org/licenses/by-nc-sa/4.0/)

Ta máxima

$\begin{array}{llllllllllll}21.7 & 21.3 & 20.6 & 21.8 & 21.2 & 24.9 & 25.0 & 26.5 & 20.4 & 23.1 & 18.6 & 19.8\end{array}$

$\left({ }^{\circ} \mathrm{C}\right)$

Ts

$\left({ }^{\circ} \mathrm{C}\right)$

$\begin{array}{llllllllllll}14.8 & 13.8 & 12.1 & 11.0 & 13.3 & 14.3 & 14.9 & 15.9 & 16.2 & 16.1 & 15.9 & 16.3\end{array}$

DPV

$(\mathrm{kPa})$

$0.19 \quad 0.36$

0.37

0.24

0.34

$0.57 \quad 0.46$

0.41

0.20

0.21

$0.17 \quad 0.15$

$P$

(mm)

$\begin{array}{llllllllllll}294.9 & 21.1 & 13.8 & 83.3 & 35.6 & 44.8 & 39.6 & 29.3 & 100.3 & 76.3 & 124.1 & 89.8\end{array}$

CAS

$\left(\mathrm{m}^{3} / \mathrm{m}^{-3}\right)$

$\begin{array}{lll}0.29 & 0.22 & 0.16\end{array}$

$\begin{array}{lll}0.16 & 0.22 & 0.16\end{array}$

0.13

0.12

0.16

$0.21 \quad 0.21$

0.22

ET

(mm)

$$
\begin{array}{llllllllllll}
93.3 & 80.0 & 68.5 & 63.5 & 72.4 & 94.0 & 93.0 & 81.7 & 71.1 & 5.5 & 85.3 & 81.9
\end{array}
$$

Donde $\mathrm{Rg}=$ radiación global; $\mathrm{Rn}=$ radiación neta; $\mathrm{RFA}=$ radiación

fotosintéticamente activa; $\mathrm{Ta}=$ temperatura del aire; $\mathrm{Ts}=$ temperatura del

suelo; $\mathrm{DPV}=$ déficit de presión de vapor; $\mathrm{P}=$ precipitación; $\mathrm{CAS}=$ contenido de agua en el suelo, y ET = evapotranspiración. 

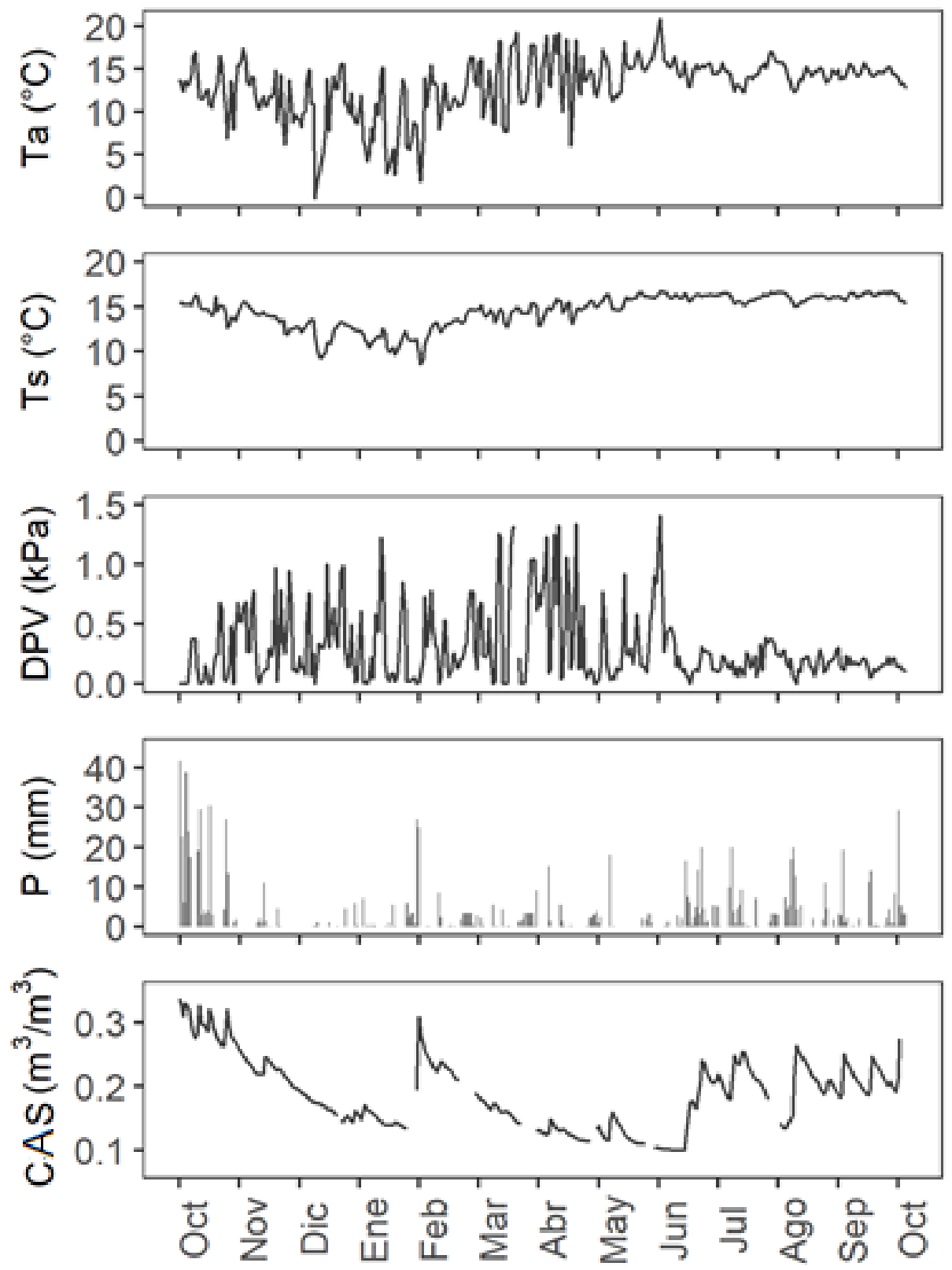

Figura 2. Variación temporal de la temperatura del aire (Ta), temperatura del suelo (Ts), déficit de presión de vapor (DPV), precipitación (P) y contenido de agua en el suelo (CAS). 


\section{Balance de energía}

El cierre de balance de energía fue de 0.81 (Figura 3), lo cual indica que hay una subestimación de los flujos de calor latente o sensible. A pesar de ello, la calidad de las mediciones de flujo fue suficiente para analizar los presupuestos de energía y evapotranspiración. El valor de cierre de energía obtenido fue similar al encontrado por Wilson et al. (2002) en los sitios FLUXNET a nivel global.

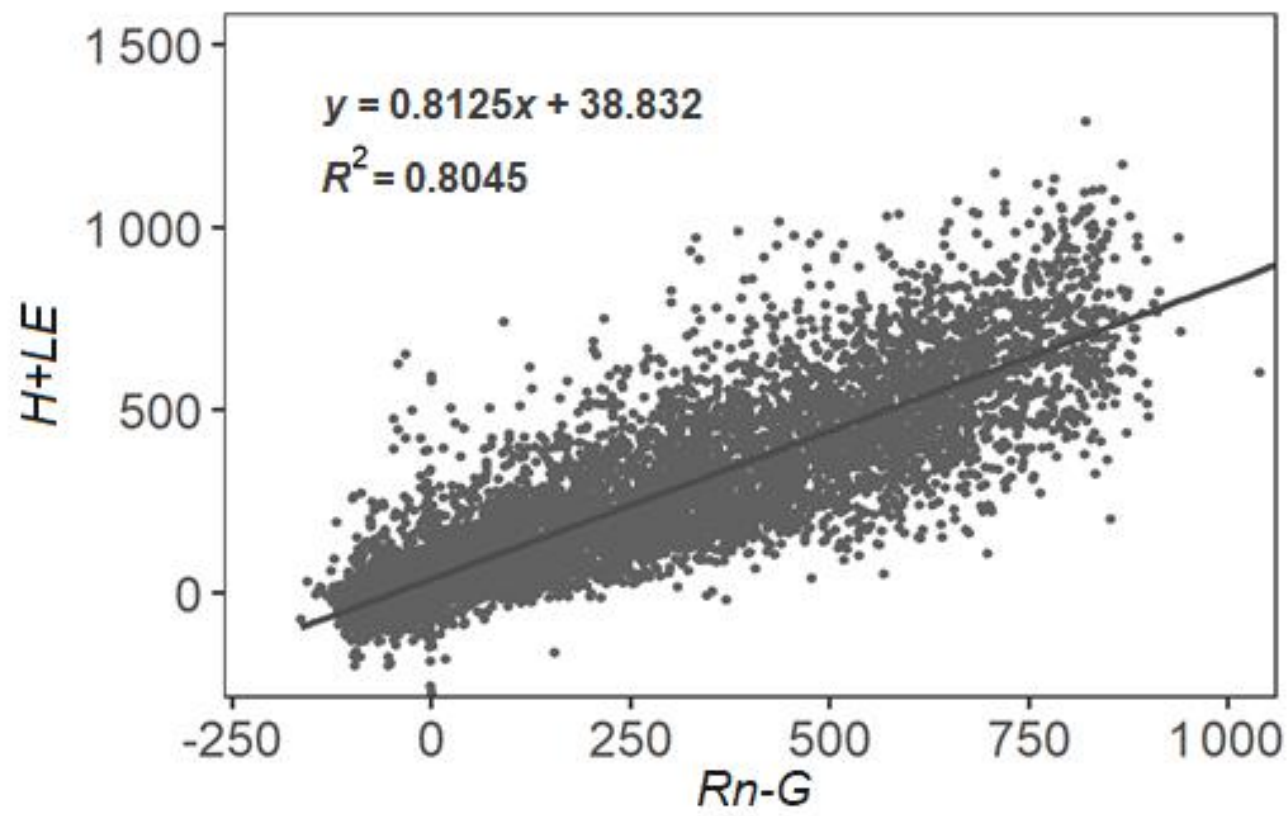

Figura 3. Dispersión de los componentes del balance de energía radiación neta $(\mathrm{Rn})$, calor del suelo $(\mathrm{G})$. calor sensible $(\mathrm{H})$ y calor latente (LE). 
El curso anual de la radiación neta, calor del suelo, calor sensible y calor latente durante el periodo de estudio se presenta en la Figura 4. La distribución de la energía disponible (radiación neta) como flujo de calor latente y calor sensible varió a lo largo del año, observándose tres tendencias. En los meses de octubre de 2017, y agosto y septiembre de 2018, cuando había más agua disponible, la principal forma de intercambio de energía estuvo representada por el calor latente.

En los meses de mayo a julio, el flujo de calor sensible fue el que dominó el intercambio de energía entre el ecosistema y la atmósfera. En el resto de los meses, el calor sensible y latente se distribuyeron equitativamente en el ecosistema. 

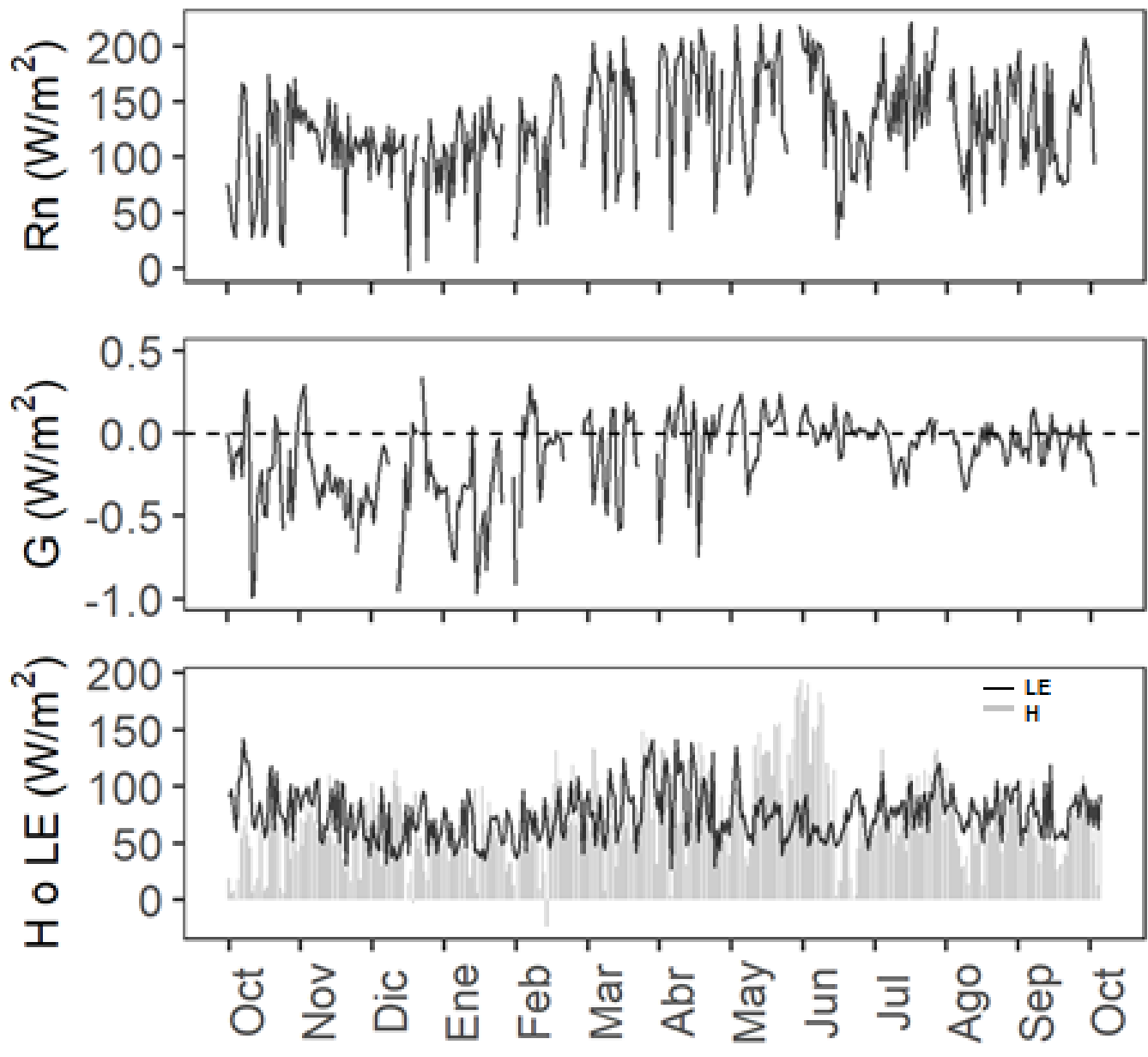

Figura 4. Variación temporal de la radiación neta $(R n)$, calor sensible $(H)$, calor latente (LE) y calor del suelo $(G)$.

La proporción de radiación neta consumida por el flujo de calor del suelo durante todo el periodo fue baja. En la Tabla 2 se presentan los valores medios mensuales de radiación neta y las proporciones utilizadas por cada flujo. 


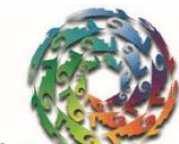

政

Ciencias $₫$ Agua
2021, Instituto Mexicano de Tecnología del Agua

Open Access bajo la licencia CC BY-NC-SA 4.0

(https://creativecommons.org/licenses/by-nc-sa/4.0/)

Tabla 2. Radiación neta y su contribución relativa para cada flujo en un bosque bajo manejo forestal.

\begin{tabular}{cccccc}
\hline Mes & Rn & LE/Rn & H/Rn & G/Rn & Relación de Bowen \\
& & & & & H/LE \\
\hline Octubre & 144.2 & 0.70 & 0.42 & -0.0015 & 0.60 \\
Noviembre & 167.5 & 0.55 & 0.53 & -0.0017 & 0.95 \\
Diciembre & 135.1 & 0.55 & 0.55 & -0.0019 & 0.99 \\
Enero & 142.6 & 0.47 & 0.52 & -0.0030 & 1.10 \\
Febrero & 157.6 & 0.51 & 0.53 & -0.0003 & 1.04 \\
Marzo & 165.0 & 0.51 & 0.50 & -0.0008 & 0.98 \\
Abril & 179.0 & 0.54 & 0.49 & -0.0001 & 0.92 \\
Mayo & 216.4 & 0.40 & 0.67 & 0.0002 & 1.65 \\
Junio & 162.9 & 0.47 & 0.55 & 0.0002 & 1.16 \\
Julio & 204.1 & 0.48 & 0.51 & -0.0003 & 1.06 \\
Agosto & 167.3 & 0.54 & 0.47 & -0.0004 & 0.86 \\
Septiembre & 179.7 & 0.52 & 0.47 & -0.0001 & 0.90 \\
\hline
\end{tabular}

Donde $\mathrm{Rn}=$ radiación neta; $\mathrm{LE}=$ flujo de calor latente; $\mathrm{H}$ = flujo de calor sensible, y $\mathrm{G}=$ flujo de calor del suelo.

\section{Evapotranspiración}


Durante el periodo de estudio, la evapotranspiración fue de $980 \mathrm{~mm}$ y excedió ligeramente la precipitación anual $(953 \mathrm{~mm})$. La evapotranspiración total mensual fue menor en enero $(63.5 \mathrm{~mm})$ y la mayor se reportó en julio $(95.5 \mathrm{~mm})$ (Tabla 1$)$. Las tasas de evapotranspiración a diferentes escalas de tiempo se muestran en la Tabla 3. La evapotranspiración media diaria varió de $2.0 \mathrm{~mm} \pm 0.6$ (enero) a $3.1 \mathrm{~mm} \pm 1.1$ (abril y julio). La tasa máxima de evapotranspiración fue de $0.86 \mathrm{~mm} \mathrm{~h}^{-1}$ y tuvo lugar en el mes de marzo. Los valores superiores de evapotranspiración máxima diaria correspondieron a los meses de marzo, abril y mayo.

Tabla 3. Tasas de evapotranspiración por hora y por día en un bosque bajo manejo forestal.

\begin{tabular}{|c|c|c|c|}
\hline Mes & $\begin{array}{l}\text { Evapotranspiración } \\
\text { máxima por hora } \\
\left(\mathrm{mm} \mathrm{h}^{-1}\right)\end{array}$ & $\begin{array}{c}\text { Evapotranspi- } \\
\text { ración media } \\
\text { diaria } \\
\left(\mathrm{mm} \text { día }^{-1} \pm \mathrm{SD}\right)\end{array}$ & $\begin{array}{l}\text { Evapotranspiración } \\
\text { máxima diaria } \\
\left(\mathrm{mm} \mathrm{dia}^{-1}\right)\end{array}$ \\
\hline Octubre & 0.74 & $3.0 \pm 0.9$ & 4.4 \\
\hline Noviembre & 0.76 & $2.7 \pm 0.8$ & 3.9 \\
\hline Diciembre & 0.76 & $2.2 \pm 0.7$ & 3.5 \\
\hline Enero & 0.77 & $2.0 \pm 0.6$ & 3.5 \\
\hline Febrero & 0.72 & $2.6 \pm 0.7$ & 3.8 \\
\hline Marzo & 0.86 & $3.0 \pm 0.9$ & 5.0 \\
\hline
\end{tabular}




$\begin{array}{cccc}\text { Abril } & 0.85 & 3.1 \pm 1.1 & 5.0 \\ \text { Mayo } & 0.83 & 2.6 \pm 0.7 & 4.8 \\ \text { Junio } & 0.70 & 2.4 \pm 0.5 & 3.4 \\ \text { Julio } & 0.71 & 3.1 \pm 0.6 & 4.4 \\ \text { Agosto } & 0.77 & 2.8 \pm 0.6 & 3.7 \\ \text { Septiembre } & 0.82 & 2.7 \pm 0.6 & 3.7\end{array}$

\section{Evapotranspiración y su respuesta a condiciones meteorológicas}

De acuerdo con el análisis de correlación de Spearman $(p<0.01)$, la radiación neta $(0.76)$ y la radiación fotosintéticamente activa (0.73) fueron las variables que tuvieron mayor relación con la evapotranspiración. La correlación fue menor, aunque significativa, con temperatura del aire $(0.42)$, humedad relativa $(-0.27$, ) y déficit de presión de vapor (0.34) (Figura 5). Los resultados del análisis de regresión lineal múltiple mostraron que la evapotranspiración estuvo más influenciada por el contenido de agua en el suelo, la radiación neta, la temperatura del aire, y el déficit de presión de vapor (Tabla 4). Para apoyar este resultado, en la Tabla 1 se puede observar que los valores más altos de evapotranspiración correspondieron con los valores promedios más altos de la radiación neta y de déficit de presión de vapor (marzo, abril y mayo). El modelo logró explicar el $61 \%$ de la variabilidad de la evapotranspiración; de acuerdo con la prueba de $F$, el modelo es 
significativo con un $P$ value $<2.2 e^{-16}$. Las correlaciones entre las variables independientes utilizadas en el modelo y el valor del factor de inflación en la varianza son indicios de que no existe relación lineal entre las variables independientes

Tabla 4. Resumen del modelo de regresión lineal múltiple.

$\begin{array}{llclll}\text { Parámetros Coeficientes } & \begin{array}{c}\text { Error } \\ \text { estándar }\end{array} & t & \operatorname{Pr}(>|t|) & \text { VIF }\end{array}$

\begin{tabular}{cccccc}
\hline Intercepto & -0.3552 & 0.0711 & -4.99 & $9.66 \mathrm{e}-07$ & \\
$\mathrm{Rn}$ & 0.0035 & 0.0003 & 11.97 & $<2 \mathrm{e}-16$ & 1.37 \\
$\mathrm{Ta}$ & 0.0316 & 0.0042 & 5.41 & $6.66 \mathrm{e}-13$ & 1.48 \\
& & & & & \\
DPV & 0.2487 & 0.0460 & 7.49 & $1.25 \mathrm{e}-07$ & 1.47 \\
& & & & & \\
CAS & 2.2001 & 0.2283 & 9.64 & $<2 \mathrm{e}-16$ & 1.14
\end{tabular}

\footnotetext{
$\mathrm{Rn}=$ radiación neta; $\mathrm{Ta}=$ temperatura del aire; $\mathrm{DPV}=$ déficit de presión de vapor; CAS = contenido de agua en el suelo.
} 
Tecnología y

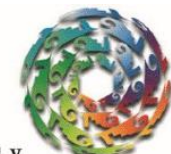

Ciencias ฐAgua
2021, Instituto Mexicano de Tecnología del Agua

Open Access bajo la licencia CC BY-NC-SA 4.0

(https://creativecommons.org/licenses/by-nc-sa/4.0/)

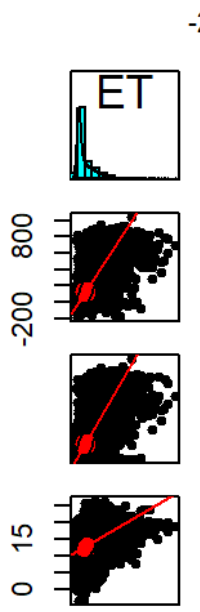

$-200800$
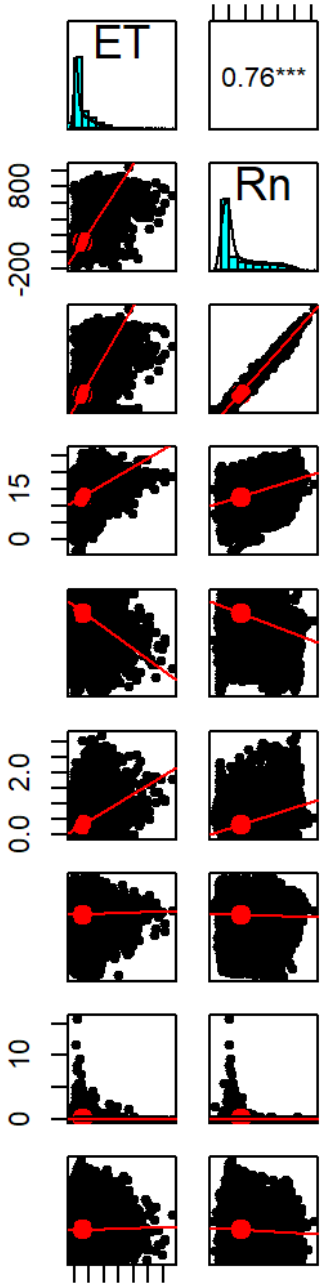

$0.0 \quad 1.0$

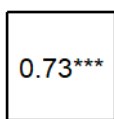

$0 \quad 15$
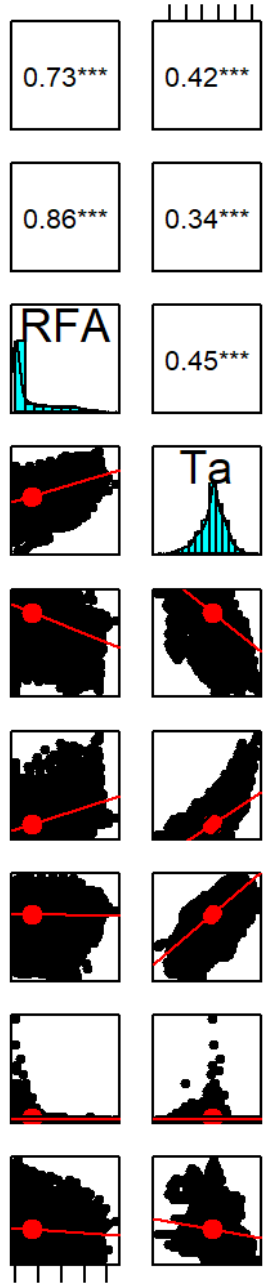

01500
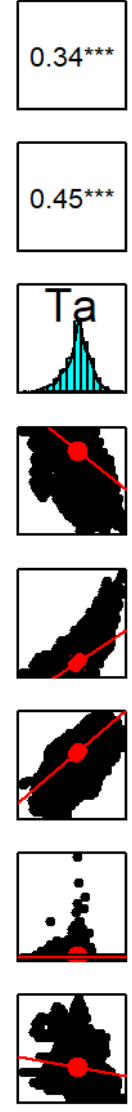

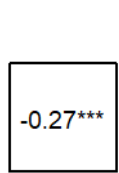

0.02 .0
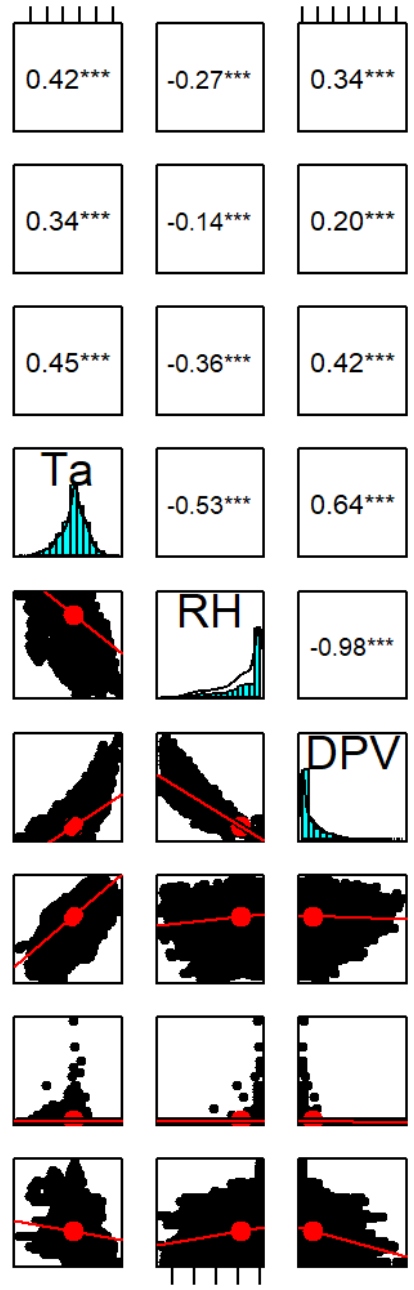

2080
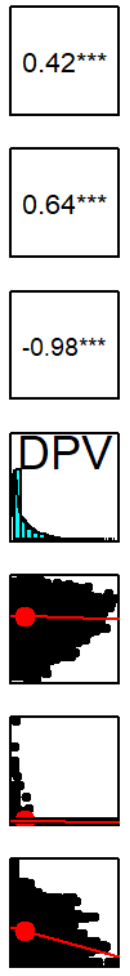
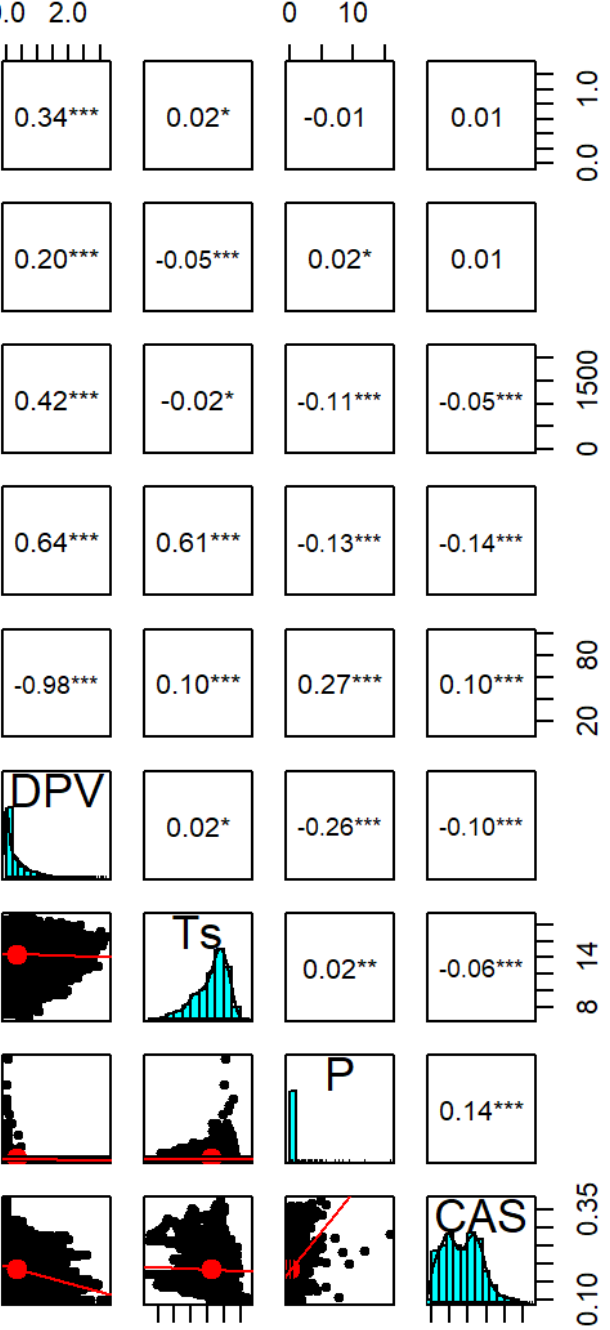

814

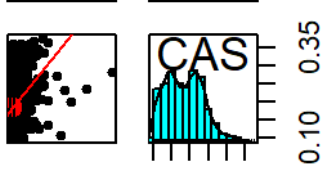

$0.10 \quad 0.35$

Figura 5. Matriz de correlación de Spearman de la evapotranspiración y variables meteorológicas. $\mathrm{ET}=$ evapotranspiración; $\mathrm{Rn}$ = radiación neta; RFA = radiación fotosintéticamente activa; $\mathrm{Ta}=$ temperatura del aire;

$\mathrm{RH}=$ humedad relativa; DPV = déficit de presión de vapor; Ts = temperatura del suelo; $\mathrm{P}=$ precipitación; CAS = contenido de agua en el suelo. 


\section{Variación estacional de la evapotranspiración}

La prueba de Kruskal-Wallis $(p<0.001)$ indicó diferencias significativas en la evapotranspiración entre las estaciones. De acuerdo con la prueba de Mann-Whitney-Wilcoxon ( $p<0.05)$, la evapotranspiración en el periodo de "nortes" fue significativamente más baja en comparación con la evapotranspiración de la época de lluvia y de seca, donde no se encontraron diferencias significativas. El mismo análisis se hizo para las variables que resultaron tener mayor influencia en la evapotranspiración. En la Tabla 5 se muestran las variaciones de evapotranspiración y las variables que resultaron tener mayor control sobre la evapotranspiración, y se resumen los resultados de las pruebas de comparación.

Tabla 5. Variación estacional de la evapotranspiración y principales factores de control.

\begin{tabular}{cccc}
\hline Variable & Nortes & Temporada & \\
& $2.4 \pm 0.7(\mathrm{~b})$ & $2.9 \pm 0.9(\mathrm{a})$ & $2.8 \pm 0.7(\mathrm{a})$ \\
\hline ET & $0.19 \pm 0.04(\mathrm{~b})$ & $0.13 \pm 0.02(\mathrm{c})$ & $0.21 \pm 0.06(\mathrm{a})$ \\
CAS & $10.4 \pm 3.69(\mathrm{~b})$ & $14.5 \pm 3.15(\mathrm{a})$ & $14.2 \pm 1.54(\mathrm{a})$ \\
Ta & $108.5 \pm 33.0(\mathrm{c})$ & $153.9 \pm 49.2(\mathrm{a})$ & $127.4 \pm 47.6(\mathrm{~b})$ \\
Rn & $0.33 \pm 0.29(\mathrm{a})$ & $0.48 \pm 0.45(\mathrm{a})$ & $0.19 \pm 0.13(\mathrm{~b})$ \\
DPV & &
\end{tabular}


Donde ET = evapotranspiración; CAS = contenido de agua en el suelo; Ta $=$ temperatura del aire; $\mathrm{Rn}=$ radiación neta, y $\mathrm{DPV}=$ déficit de presión de vapor. Letras iguales indican que no hubo diferencias significativas ( $p$ $<0.05)$ entre las temporadas.

\section{Discusión}

\section{Balance de energía}

El manejo forestal y la disponibilidad de humedad en el suelo tienen un control importante en la división de la energía (Gholz \& Clark, 2002; Sun et al., 2010). En los bosques templados, durante la temporada de crecimiento, la disponibilidad de humedad en el suelo no suele ser limitante; por lo que, durante este periodo, es común que el flujo de calor latente sea superior que el calor sensible (Yan et al., 2017). En este estudio, sólo en los meses de junio, agosto, septiembre y octubre el calor latente tuvo valores superiores al calor sensible. Varios autores relacionan 
estas dos variables energéticas a través de la Relación de Bowen (H/LE) (Gholz \& Clark, 2002; Giambelluca et al., 2009; Tudoroiu et al., 2018).

Se encontró que para este sitio la Relación de Bowen fue menor en octubre (0.60) y un valor mayor se obtuvo para mayo (1.65) (Tabla 2). En este mes, los promedios mensuales de radiación neta y temperatura máxima fueron superiores, y el contenido de agua en el suelo fue menor. Es posible que bajo estas condiciones, el arbolado se estrese y en consecuencia genere modificaciones significativas en la productividad del ecosistema. Sin embargo, esto dependerá de los mecanismos de respuesta y tolerancia que las plantas hayan desarrollado ante tales situaciones (Méndez-Espinoza \& Vallejo-Reyna, 2019). Por lo general, el cierre de las estomas es el principal mecanismo de respuesta ante el déficit hídrico y altas temperaturas. Si únicamente las temperaturas altas son el problema, el arbolado continuará transpirando, ya que debido a su efecto de enfriamiento mantendrá una temperatura de la superficie foliar adecuada (Harfouche, Meilan, \& Altman, 2014). Probablemente esto es lo que suceda en el sitio de estudio, dado que no se encontraron diferencias significativas en las tasas de evapotranspiración del periodo de lluvia y la temporada de secas.

También se encontró que el flujo de calor del suelo fue bajo, esto es una característica común en suelos bajo una cubierta forestal perennifolia y se ve reflejado en variaciones reducidas en la temperatura del suelo.

\section{Evapotranspiración}


Los resultados de este estudio mostraron que la evapotranspiración del bosque bajo manejo fue variable durante el periodo de estudio. La evapotranspiración anual (980 mm) fue similar a las reportadas en una plantación de Pinus taeda L. (1 $076 \pm 104$ mm/año) en Carolina del Norte, EUA (Sun et al., 2010). En general, estos sitios presentaron rangos de temperatura similares; no tuvieron problema en la disponibilidad de agua, y el suelo tuvo un alto contenido de materia orgánica. De acuerdo con evaluaciones de las tasas de evapotranspiración asumidas para bosques templados ( $710.8 \mathrm{~mm}$ promedio), éstas son más bajas que en bosques tropicales (1 502.2 mm) (Levia, Carlyle-Moses, \& Tanaka, 2011). En el caso de las regiones áridas y semiáridas, la magnitud de la evapotranspiración es mucho menor que las reportadas en este estudio. En tal tipo de ecosistemas, uno de los principales factores que influye en la dinámica de la evapotranspiración es la precipitación y además existe una clara influencia de la vegetación (Bierderman et al., 2016). En condiciones de sequía, el proceso de transpiración se ve limitado a causa del estrés hídrico en las plantas por la alta demanda de evaporación atmosférica y el contenido restringido de agua del suelo (Meza, Montes, Bravo-Martínez, Serrano-Ortiz, \& Kowalski, 2018).

Los procesos que controlan la evapotranspiración implican los efectos combinados de factores físicos y biológicos (Novick et al., 2015) controlados por las condiciones ambientales (Xu et al., 2014). Por lo general, en sitios ubicado en latitudes medias y altas, la radiación neta y la temperatura del aire son las variables que dominan en el control de la 
Tecnologíay

Ciencias $₫$ Agua
2021, Instituto Mexicano de Tecnología del Agua

Open Access bajo la licencia CC BY-NC-SA 4.0

(https://creativecommons.org/licenses/by-nc-sa/4.0/)

evapotranspiración (Chen, Xue, \& Hu, 2018). Esto se debe a que la radiación es considerada la dotación de energía disponible para llevar a cabo la evaporación (Izadifar \& Elshorbagy, 2010); además, la capacidad de la atmósfera para retener agua se incrementa conforme aumenta la temperatura del aire (Pan et al., 2015; Wagle et al., 2016). Sin embargo, cuando la humedad en el suelo es limitante, aumenta el déficit de presión de vapor, lo que afecta la respuesta fisiológica de las plantas. Por lo tanto, el déficit de presión de vapor se vuelve más relevante en el control de la evapotranspiración (Zha et al., 2013). Cuando la humedad del suelo no es la suficiente, se esperaría que la planta cerrará las estomas para evitar la pérdida excesiva de agua (Landsberg \& Sands, 2011); pero las respuestas de las plantas a las condiciones climáticas dependen de las especies, y del historial de vida y manejo; por lo tanto, puede haber un componente genético en las respuestas climáticas que haga que tengan respuestas fisiológicas diferenciales a estímulos similares (Qaderi, Martel, \& Dixon, 2019). El papel de la vegetación ha sido evaluado a través del monitoreo del Índice de Área Foliar, conductancia de la hoja o del dosel, o índices de vegetación de diferencia normalizada (NDVI, por sus siglas en inglés) (Cabral, Da-Rocha, Gash, Freitas, \& Ligo, 2015; Mo et al., 2018; Yang et al., 2017); pero pocos son los estudios que evalúan los efectos de los factores ambientales en las estructuras encargadas del transporte de agua, en especial el xilema (Qaderi et al., 2019).

En una plantación dominada por Pinus tabuliformis, en Beijing China, un bajo contenido de agua en el suelo $\left(0.1 \mathrm{~m}^{3} / \mathrm{m}^{3}\right)$, en combinación con una radiación neta $\left(\sim 200 \mathrm{~W} / \mathrm{m}^{2}\right)$ y temperaturas altas $\left(\sim 25^{\circ}\right)$, causaron una evapotranspiración baja ( $51.7 \mathrm{~mm}$, en el mes más seco) (Ma et al., 2018). En contraste, aquí se reporta que las tasas de 


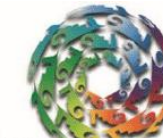

Ciencias $\overline{\text { Tagua }}$

evapotranspiración más altas ( $89.5 \mathrm{~mm} / \mathrm{mes,} \mathrm{en} \mathrm{promedio),} \mathrm{ocurrieron}$ en la época de secas (Tabla 1). Esto sugiere que durante este periodo, la vegetación no tuvo restricciones de suministro de agua y que tal vez la conductancia del dosel no sea un factor que limite el proceso de evapotranspiración. Las condiciones de disponibilidad de humedad reducen la sensibilidad de la conductancia del dosel a los cambios en el DPV (Liu et al., 2018). Además, las variables climáticas lograron explicar la mayor parte $(61 \%)$ de la variabilidad de la evapotranspiración. El nulo problema de falta de agua —durante la época de secas_ puede atribuirse a la capacidad de los árboles para tener acceso a fuentes de agua más profundas, como lo señalan Thompson et al. (2011). Otra posibilidad es que los eventos de precipitación durante el periodo de "nortes" proporcionaron humedad suficiente para contrarrestar los impactos de la época de secas.

Los efectos del manejo forestal en la evapotranspiración no se evaluaron. Sin embargo, un aclareo con una intensidad de $50 \%$ realizado en una plantación de Pinus taeda L. mostró pequeñas variaciones interanuales en la radiación neta, y una ligera disminución de la evapotranspiración. Con base en los resultados, Liu et al. (2018) sugirieron que los bosques eran relativamente insensibles a las actividades de manejo, pues el rápido crecimiento del sotobosque y el cierre del dosel permitieron una pronta recuperación de las tasas de evapotranspiración. Los efectos del manejo forestal se vuelven más notables durante los periodos secos en sistemas con agua limitada, pero tienden a disminuir conforme la edad del rodal aumenta (Sun et al., 2010; Yang et al., 2017). 
Los hallazgos aquí mostrados representan información sobre los principales factores climáticos que controlaron el intercambio de agua en un bosque bajo manejo. Estos resultados se basaron en el monitoreo continuo a lo largo de un año. Durante este periodo, la precipitación fue $20 \%$ menor a la ocurrida en años anteriores. Puede ser que estas variaciones hayan provocado cambios en la evapotranspiración. No obstante, Zha et al. (2013) señalan que las variaciones interanuales en la evapotranspiración suelen ser menores en bosques de coníferas (7-14\%) en comparación con los bosques caducifolios, donde se han reportado rangos interanuales de $\sim 30 \%$. A pesar de ello, son necesarios estudios a largo plazo, que además involucren otros parámetros, que permitan aclarar y ampliar la comprensión de los procesos de intercambio de agua en los bosques templados mexicanos.

\section{Conclusiones}

La mayor contribución de la radiación neta al flujo de calor sensible se observó en periodos donde la temperatura fue superior y el contenido de agua en el suelo bajo. El flujo de calor latente fue mayor que el calor sensible, principalmente durante la temporada de lluvia. 
La evapotranspiración estuvo controlada por el contenido de agua en el suelo, la temperatura, la radiación neta, y el déficit de presión de vapor. En conjunto, estos factores explicaron el $61 \%$ de la variabilidad de la evapotranspiración. La medición y el análisis de otros parámetros, como índice de área foliar, conductancia del dosel, potencial hídrico y humedad del suelo a diferentes profundidades pueden aclarar y ampliar la comprensión de este proceso.

La evapotranspiración total fue de $980 \mathrm{~mm}$. Valores mínimos de evapotranspiración ocurrieron durante el periodo denominado como "nortes". A pesar de los cambios estacionales en la radiación neta y el contenido de agua en el suelo, no se encontraron diferencias significativas entre la evapotranspiración ocurrida durante la temporada de lluvia y la época de secas.

Las mediciones del intercambio de energía y vapor de agua reportadas en este estudio representan las primeras observaciones continuas realizadas en un bosque bajo manejo forestal en México. Esta información permitió caracterizar los patrones de distribución de la radiación y la variabilidad de la evapotranspiración.

\section{Agradecimientos}

Este estudio fue financiado por el Programa de Paisajes Sostenibles de la Agencia para el Desarrollo Internacional de los Estados Unidos de América, a través de la Oficina de Programas Internacionales del Servicio Forestal de la USDA, proyecto "Reducing Greehouse Gas Emissions and Improving Forest Managemente in Mexico" (Acuerdo No. 12-IJ- 


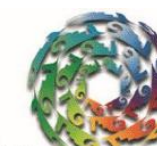

Tiencialias

11242306-033). También fue financiado por el Consejo Nacional de Ciencia y Tecnología de México, a través del proyecto "El papel de los bosques bajo gestión forestal comunitaria en la mitigación del cambio climático" (PN 2017-6231)

\section{Referencias}

Aguirre-Salado, C. A., Valdez-Lazalde, J. R., Ángeles-Pérez, G., De Los Santos-Posadas, H. M., Haapanen, R., \& Aguirre-Salado, A. I. (2009). Mapping aboveground tree carbon in managed Patula pine forests in Hidalgo, México. Agrociencia, 43, 209-220.

Alvarado-Barrientos, M. S., Holwerda, F., Asbjornsen, H., Dawson, T. E., \& Bruijnzeel, L. A. (2014). Suppression of transpiration due to cloud immersion in a seasonally dry Mexican weeping pine plantation. Agricultural and Forest Meteorology, 186, 12-25. DOI: 10.1016/j.agrformet.2013.11.002

Ángeles-Pérez, G., Méndez-López, B., Valdez-Lazalde, R., PlascenciaEscalante, F. O., De-Los-Santos-Posadas, H. M., Chávez-Aguilar, G., \& Maldonado-Montero, V. (2015). Estudio de Caso del Sitio de Monitoreo Intensivo del Carbono en Hidalgo. Montecillo, México: Proyecto México-Noruega-Colegio de Postgraduados.

Aubinet, M., Vesala, T., \& Papale, D. (2012). Eddy Covariance: A Practical Guide to Measurement and Data Analysis. DOI: 10.1007/978-94007-2351-1. New York, USA: Springer.

Baldocchi, D. D. (2003). Assessing the eddy covariance technique for evaluating carbon dioxide exchange rates of ecosystems: Past, 
present and future. Global Change Biology, 9(4), 479-492. DOI: 10.1046/j.1365-2486.2003.00629.x

Baldocchi, D. D. (2019). How eddy covariance flux measurements have contributed to our understanding of Global Change Biology. Global Change Biology, 26, 242-260. DOI:10.1111/gcb.14807

Berry, Z. C., Gotsch, S. G., Holwerda, F., Muñoz-Villers, L. E., \& Asbjornsen, H. (2016). Slope position influences vegetationatmosphere interactions in a tropical montane cloud forest. Agricultural and Forest Meteorology, 221, 207-218. DOI: 10.1016/j.agrformet.2016.02.012

Biederman, J. A., Scott, R. L., Goulden, M. L., Vargas, R., Litvak, M. E., Kolb, T. E., Yepez, E. A., Oechel, W. C., Blanken, P. D., Bell, T. W., Garatuza-Payan, J., Maurer, G. E., Dore, S., \& Burns, S. P. (2016). Terrestrial carbon balance in a drier world: The effects of water availability in southwestern North America. Global Change Biology, 22, 1867-1879. DOI: $10.1111 /$ gcb.13222

Bonan, G. (2016). Ecological climatology: Concepts and applications. (3 ${ }^{\text {rd }}$ ed.). Boulder \& New York, USA: Center for Atmospheric Research / Cambridge University Press.

Brümmer, C., Black, T. A., Jassal, R. S., Grant, N. J., Spittlehouse, D. L., Chen, B., Nesic. Z., Amiro, B. D., Arain, M. A., Barr, A. Bourqueg, C. P., Coursolle, C., Dunn, A. L., Flanagan, L. B., Humphreysk, E. R., Lafleur, P. M., Margolish, H. A., McCaughey, J. H., \& Wofsyn, S. C. (2012). How climate and vegetation type influence evapotranspiration and water use efficiency in Canadian forest, 


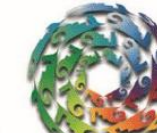

Tienciass

peatland and grassland ecosystems. Agricultural and Forest Meteorology, 153, 14-30. DOI: 10.1016/j.agrformet.2011.04.008

Burba, G. (2013). Eddy covariance method: For scientific, industrial, agricultural, and regulatory applications. Lincoln, USA: LI-COR Biosciences.

Cabral, O. M. R., Da-Rocha, H. R., Gash, J. H., Freitas, H. C., \& Ligo, M. A. V. (2015). Water and energy fluxes from a woodland savanna (cerrado) in southeast Brazil. Journal of Hydrology: Regional Studies, 4(PB), 22-40. DOI: 10.1016/j.ejrh.2015.04.010

Canny, M. J. (1998). Transporting water in plants that possible by protecting the stretched water and repairing it when it breaks. American Scientist, 86(2), 152-159.

Chen, Y., Xue, Y., \& Hu, Y. (2018). How multiple factors control evapotranspiration in North America evergreen needleleaf forests. Science of the Total Environment, 622-623, 1217-1224. DOI: 10.1016/j.scitotenv.2017.12.038

Conafor, Comisión Nacional Forestal. (2012). Manual y procedimientos para el muestreo de campo. En: Inventario nacional forestal y de suelos. México, DF, México: Comisión Nacional Forestal.

Cristiano, P. M., Campanello, P. I., Bucci, S. J., Rodriguez, S. A., Lezcano, O. A., Scholz, F. G., Madanes, N., Di Francescantonio, D., Carrasco, L. O., Zhang, Y. J., \& Goldstein, G. (2015). Evapotranspiration of subtropical forests and tree plantations: A comparative analysis at different temporal and spatial scales. Agricultural and Forest Meteorology, 203, 96-106. DOI: 10.1016/j.agrformet.2015.01.007 
Delgado-Balbuena J., Yépez, E. A., Paz-Pellat, F., Ángeles-Pérez, G., Alvarado-Barrientos, M. S., Bullock, S. H., ... \& Watts, C. J. (2019). Flujos verticales de carbono en ecosistemas terrestres. En: PazPellat, F., Hernández-Ayon, J. M., Sosa-Avalos, R., \& VelázquezRodríguez, A. S. (eds.). Estado del ciclo del carbono en México Agenda Azul y Verde (pp. 605-625). Texcoco, México: Programa Mexicano del Carbono.

Dolman, A. J., Moors, E. J., Grundwald, P. B., \& Bernhofer, C. (2003). Factors controlling forest atmosphere exchange of water, energy, and carbon. In: Valentini, R. (ed.). Fluxes of carbon, water and energy of European forests. Ecological studies (analysis and synthesis) (pp. 207-224). Berlin \& Heidelberg, Germany: Springer. DOI: $10.1007 / 978-3-662-05171-9 \_6$

Ellison, D., Morris, C. E., Locatelli, B., Sheil, D., Cohen, J., Murdiyarso, D.,... \& Sullivan, C. A. (2017). Trees, forests and water: Cool insights for a hot world. Global Environmental Change, 43, 51-61. DOI: 10.1016/j.gloenvcha.2017.01.002

Falge, E., Baldocchi, D. D., \& Olson, R. (2001). Gap filling strategies for defensible annual sums of net ecosystem exchange. Agricultural and Forest Meteorology, 107, 43-69. DOI: 10.1016/S01681923(00)00225-2

García, A. G., Campos, A. N., Di Bella, C. M., \& Posse, G. (2008). Evolución de la evapotranspiración en diferentes coberturas vegetales de la Argentina utilizando productos derivados del sensor MODIS. Buenos Aires, Argentina: Instituto Nacional de Tecnología Agropecuaria. 
Gholz, H. L., \& Clark, K. L. (2002). Energy exchange across a chronosequence of slash pine forests in Florida. Agricultural and Forest Meteorology, 112(2), 87-102. DOI: 10.1016/S01681923(02)00059-X

Giambelluca, T. W., Martin, R. E., Asner, G. P., Huang, M., Mudd, R. G., Nullet, M. A.,... \& Foote, D. (2009). Evapotranspiration and energy balance of native wet montane cloud forest in Hawai'i. Agricultural and Forest Meteorology, 149(2), 230-243. DOI: 10.1016/j.agrformet.2008.08.004

Harfouche, A., Meilan, R., \& Altman, A. (2014). Molecular and physiological responses to abiotic stress in forest trees and their relevance to tree improvement. Tree Physiology, 34, 1181-1198. DOI: $10.1093 /$ treephys/tpu012.

Hollinger, D. Y. (2008). Defining a landscape-scale monitoring tier for the North American Carbon Program. In: Hoover, C. M. (ed.). Field Measurements for Forest Carbon Monitoring (pp. 3-16). Durham, USA. DOI: $10.1007 / 978-1-4020-8506-2 \_1$

Holwerda, F., Alvarado-Barrientos, M. S., \& González-Martínez, T. M. (2016). Surface energy exchange in a tropical montane cloud forest environment: Flux partitioning, and seasonal and land cover-related variations. Agricultural and Forest Meteorology, 228-229, 13-28. DOI: $10.1016 /$ j.agrformet.2016.06.011

Huryna, H., \& Pokorný, J. (2016). The role of water and vegetation in the distribution of solar energy and local climate: A review. Folia Geobot, 51(3), 191-208. DOI: 10.1007/s12224-016-9261-0 


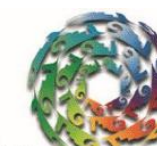

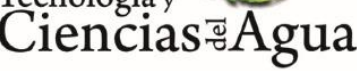

2021, Instituto Mexicano de Tecnología del Agua

Open Access bajo la licencia CC BY-NC-SA 4.0

(https://creativecommons.org/licenses/by-nc-sa/4.0/)

Izadifar, Z., \& Elshorbagy, A. (2010). Prediction of hourly actual evapotranspiration using neural networks, genetic programming, and statistical models. Hydrological Processes, 24(23), 3413-3425. DOI: 10.1002/hyp. 7771

Kljun, N., Calanca, P., Rotach, M. W., \& Schmid, H. P. (2015). A simple two-dimensional parameterisation for Flux Footprint Prediction (FFP). Geoscientific Model Development, 8, 3695-3713. DOI: 10.5194/gmd-8-3695-2015

Landsberg, J., \& Sands, P. (2011). Physiological Ecology of Forest Production. USA. DOI: 10.1016/S1936-7961(11)04007-3

Law, B. E., Falge, E., Gu, L., Baldocchi, D. D., Bakwin, P., Berbigier, P.,... \& Wofsy, S. (2002). Environmental controls over carbon dioxide and water vapor exchange of terrestrial vegetation. Agriculture and Forest Meteorology, 113(113), 97-120.

Levia, D. F., Carlyle-Moses, D., \& Tanaka, T. (2011). Forest hydrology and biogeochemistry: Synthesis of past research and future directions. Analysis and synthesis, (216), 407-423. DOI: 10.1007/978-94-007-1363-5

LI-COR, Inc. (2016). EddyPro ${ }^{\circledR}$ Software (Version 6.2). Recuperado de https://www.licor.com/env/support/EddyPro/software.html

Liu, X., Sun, G., Mitra, B., Noormets, A., Gavazzi, M. J., Domec, J.,... \& Mcnulty, S. G. (2018). Drought and thinning have limited impacts on evapotranspiration in a managed pine plantation on the southeastern United States coastal plain. Agricultural and Forest Meteorology, 262, 14-23. DOI: $10.1016 /$ j.agrformet.2018.06.025 
Liu, Y., \& El-Kassaby, Y. A. (2018). Evapotranspiration and favorable growing degree-days are key to tree height growth and ecosystem functioning: Meta-analyses of Pacific Northwest historical data. Nature, 8228 (8), 1-12. DOI: 10.1038/s41598-018-26681-1

Ma, J., Zha, T., Jia, X., Tian, Y., Bourque, C. P. A., Liu, P.,... \& Chen, W. (2018). Energy and water vapor exchange over a young plantation in northern China. Agricultural and Forest Meteorology, 263, 334345. DOI: $10.1016 /$ j.agrformet.2018.09.004

Martínez-Yrízar, A., Álvarez-Sánchez, J., \& Maass, M. (2017). Análisis y perspectivas del estudio de los ecosistemas terrestres de México: dinámica hidrológica y flujos de nitrógeno y fósforo. Revista Mexicana de Biodiversidad, 88, 27-41. DOI: 10.1016/j.rmb.2017.10.008

Matsumoto, K., Ohta, T., Nakai, T., Kuwada, T., Daikoku, K., Iida, S.,... \& Hattori, S. (2008). Energy consumption and evapotranspiration at several boreal and temperate forests in the Far East. Agricultural and Forest Meteorology, 148(12), 1978-1989. DOI: 10.1016/j.agrformet.2008.09.008

Mauder, M., \& Foken, T. (2006). Impact of post-field data processing on eddy covarianceflux estimates and energy balance closure. Meteorologische Zeitschrift, 15(6), 597-609

Méndez-Espinoza, C., \& Vallejo-Reyna, M. Á. (2019). Mecanismos de respuesta al estrés abiótico: hacia una perspectiva de las especies forestales. Revista Mexicana de Ciencias Forestales, 10(56), 33-64. DOI: $10.29298 /$ rmcf.v10i56.567

Meza, F. J., Montes, C., Bravo-Martínez, F., Serrano-Ortiz, P., \& Kowalski, A. S. (2018). Soil water content effects on net ecosystem $\mathrm{CO}_{2}$ 
exchange and actual evapotranspiration in a Mediterranean semiarid savanna of Central Chile. Scientific Reports, 8(1), 1-11. DOI: $10.1038 / s 41598-018-26934-z$

Miyazaki, S., Ishikawa, M., Baatarbileg, N., Damdinsuren, S., Ariuntuya, N., \& Jambaljav, Y. (2014). Interannual and seasonal variations in energy and carbon exchanges over the larch forests on the permafrost in northeastern Mongolia. Polar Science, 8(2), 166-182. DOI: $10.1016 / j$.polar.2013.12.004

Mo, X., Liu, S., Chen, X., \& Hu, S. (2018). Variability, tendencies, and climate controls of terrestrial evapotranspiration and gross primary productivity in the recent decade over China. Ecohydrology, 11(4), 1-13. DOI: $10.1002 /$ eco. 1951

Monarrez-González, J. C., Perez-Verdin, G., López-González, C., MárquezLinarez, M. A., \& González-Elizondo, M. del S. (2018). Efecto del manejo forestal sobre algunos servicios ecosistémicos en los bosques templados de México. Madera y Bosques, 24(2), 1-16. DOI: $10.21829 /$ myb.2018.2421569

Moncrieff, J. B. (2004). Averaging, detrending and filtering of eddy covariance time series. In: Lee, X., Massman, W. J., \& Law, B. E. (eds.). Handbook of micrometeorology: A guide for surface flux measurements. Dordrecht, The Netherlands: Kluwer Academic.

Moncrieff, J. B. (1997). A system to measure surface fluxes of momentum sensible heat, water vapor and carbon dioxide. Journal of Hydrology, 589-611.

Novick, K. A., Oishi, A. C., Ward, E. J., Siqueira, M. B. S., Juang, J. Y., \& Stoy, P. C. (2015). On the difference in the net ecosystem exchange 
of $\mathrm{CO}_{2}$ between deciduous and evergreen forests in the southeastern United States. Global Change Biology, 21(2), 827-842. DOI: $10.1111 / \mathrm{gcb} .12723$

Özhan, S., Gökbulak, F., Serengil, Y., \& Özcan, M. (2010). Evapotranspiration from a mixed deciduous forest ecosystem. Water Resources Management, 24(10), 2353-2363. DOI: 10.1007/s11269009-9555-6

Pan, S., Tian, H., Dangal, S. R. S., Yang, Q., Yang, J., Lu, C.,... \& Ouyang, Z. (2015). Responses of global terrestrial evapotranspiration to climate change and increasing atmospheric $\mathrm{CO}_{2}$ in the 21st century. Earth's Future, 3(1), 15-35. DOI: 10.1002/2014EF000263

Pérez-Ruiz, E. R., Garatuza-Payan, J., Watts, C. J., Rodriguez, J. C., Yepez, E. A., \& Scott, R. L. (2010). Carbon dioxide and water vapour exchange in a tropical dry forest as influenced by the North American Monsoon System (NAMS). Journal of Arid Environments, 74(5), 556563. DOI: $10.1016 /$ j.jaridenv.2009.09.029

Perez-Verdin, G., Monarrez-González, J. C., Tecle, A., \& Pompa-Garcia, M. (2018). Evaluating the multi-functionality of forest ecosystems in northern Mexico. Forests, 9(4), 1-14. DOI: 10.3390/f9040178

Qaderi, M. M., Martel, A. B., \& Dixon, S. L. (2019). Environmental factors influence plant vascular system and water regulation. Plants, 8(3), 1-23. DOI: $10.3390 /$ plants 8030065

R Core Team. (2018). R: A language and environment for statistical computing. R Foundation for Statistical Computing. Vienna, Austria. Recuperado de http://www.R-project.org/ 
Reichstein, M., Falge, E., Baldocchi, D., Papale, D., Aubinet, M., Berbigier, P.,... \& Valentini, R. (2005). On the separation of net ecosystem exchange into assimilation and ecosystem respiration: Review and improved algorithm. Global Change Biology, 11(9), 1424-1439. DOI: 10.1111/j.1365-2486.2005.001002.x

Sanwangsri, M., Hanpattanakit, P., \& Chidthaisong, A. (2017). Variations of energy fluxes and ecosystem evapotranspiration in a young secondary dry dipterocarp forest in Western Thailand. Atmosphere, 8(8). DOI: $10.3390 /$ atmos 8080152

Soriano-Luna, M. de los Á., Ángeles-Pérez, G., Guevara, M., Birdsey, R., Pan, Y., Vaquera-Huerta, H., ... \& Vargas, R. (2018). Determinants of above-ground biomass and its spatial variability in a temperate forest managed for timber production. Forests, 9(8), 1-20. DOI: $10.3390 /$ f9080490

Sun, G., Noormets, A., Gavazzi, M. J., Mcnulty, S. G., Chen, J., Domec, J.,... \& Skaggs, R. W. (2010). Energy and water balance of two contrasting loblolly pine plantations on the lower coastal plain of North Carolina, USA. Forest Ecology and Management, 259, 12991310. DOI: $10.1016 /$ j.foreco.2009.09.016

Sun, G., Domec, J. C., \& Amatya, D. M. (2016). Forest evapotranspiration: Measurement and modelling at multiple scales. In: Forest hydrology: Processes, management and assessment (pp. 32-50). DOI: $10.1079 / 9781780646602.0032$

Sun, X., Zou, C. B., Wilcox, B., \& Stebler, E. (2018). Effect of vegetation on the energy balance and evapotranspiration in tallgrass prairie: $A$ 
Paired study using the eddy-covariance method. Boundary-Layer Meteorology. DOI: 10.1007/s10546-018-0388-9

Thompson, S. E., Harman, C. J., Konings, A. G., Sivapalan, M., Neal, A., \& Troch, P. A. (2011). Comparative hydrology across Ameriflux sites: The variable roles of climate, vegetation, and groundwater. Water Resources Research, 47(7), 1-17. DOI: 10.1029/2010WR009797

Tsuruta, K., Kosugi, Y., Takanashi, S., \& Tani, M. (2016). Inter-annual variations and factors controlling evapotranspiration in a temperate Japanese cypress forest. Hydrological Processes, 30(26), 5012-5026. DOI: $10.1002 /$ hyp. 10977

Tudoroiu, M., Genesio, L., Gioli, B., Schume, H., Knohl, A., Brümmer, C., \& Miglietta, F. (2018). Solar dimming above temperate forests and its impact on local climate. Environmental Research Letters, 13(6). DOI: $10.1088 / 1748-9326 /$ aac4e0

Ussiri, D. A. N., \& Lal, R. (2017). Carbon sequestration for climate change mitigation and adaptation. Springer, USA. DOI: 10.1007/978-3-31953845-7

Vickers, D., \& Mahrt, L. (1997). Quality control and flux sampling problems for tower and aircraft data. Journal of Atmospheric and Oceanic Technology, 14, 512-526.

Wagle, P., Xiao, X., Kolb, T. E., Law, B. E., Wharton, S., Monson, R. K.,... \& Gowda, P. H. (2016). Differential responses of carbon and water vapor fluxes to climate among evergreen needleleaf forests in the USA. Ecological Processes, 5(1). DOI: 10.1186/s13717-016-0053-5

Webb, E. K., Pearman, G. I., \& Leuning, R. (1980) Correction of flux measurements for density effects due to heat and water vapour 
transfer. Quarterly Journal of the Royal Meteorological Society, 106, 85-100.

Wilczak, J. M., Oncley, S. P., \& Stage, S. A. (2001). Sonic anemometer tilt correction algorithms. Boundary-Layer Meteorology, 99, 127-150 DOI: $10.1023 / A: 1018966204465$

Wilson, K. B., \& Baldocchi, D. D. (2000). Seasonal and interannual variability of energy fluxes over a broadleaved temperate deciduous forest in North America. Agricultural and Forest Meteorology, 102, 83-103.

Wilson, K., Goldstein, A., Falge, E., Aubinet, M., Baldocchi, D., Berbigier, P.,... \& Verma, S. (2002). Energy balance closure at FLUXNET sites. Agricultural and Forest Meteorology, 113, 223-243.

Wutzler, T., Lucas-Moffat, A., Migliavacca, M., Knauer, J., Sickel, K., Šigut, L.,... \& Reichstein, M. (2018). Basic and extenx|sible postprocessing of eddy covariance flux data with REddyProc. Biogeosciences, 15, 5015-5030. DOI:10.5194/bg-15-5015-2018

Xu, M., Wen, X., Wang, H., Zhang, W., Dai, X., Song, J.,... \& Yu, G. (2014). Effects of climatic factors and ecosystem responses on the interannual variability of evapotranspiration in a coniferous plantation in subtropical China. PLOS ONE, 9(1), 1-14. DOI: 10.1371/journal.pone.0085593

Yan, C., Zhao, W., Wang, Y., Yang, Q., Zhang, Q., \& Qiu, G. Y. (2017). Effects of forest evapotranspiration on soil water budget and energy flux partitioning in a subalpine valley of China. Agricultural and Forest Meteorology, 246, 207-217. DOI: 10.1016/j.agrformet.2017.07.002 
Yang, Y., Anderson, M. C., Gao, F., Hain, C. R., Semmens, K. A., Kustas, W. P.,... \& Sun, G. (2017). Daily Landsat-scale evapotranspiration estimation over a forested landscape in North Carolina, USA, using multi-satellite data fusion. Hydrology and Earth System Sciences, 21(2), 1017-1037. DOI: 10.5194/hess-21-1017-2017

Zha, T., Li, C., Kellomaki, S., Peltola, H., Wang, K., \& Zhang, Y. (2013). Controls of evapotranspiration and $\mathrm{CO}_{2}$ Fluxes from Scots pine by surface conductance and abiotic factors. PLOS ONE, 8(7). DOI: 10.1371/journal.pone.0069027 